\title{
To boldly go - or to go too boldly? The accelerator hypothesis revisited
}

\author{
E. A. M. Gale
}

Published online: 20 June 2007

(C) Springer-Verlag 2007

\section{There's many a beautiful hypothesis slain by an ugly fact}

\section{T. H. Huxley}

Biological science notoriously leads us into a world of increasing complexity, and concepts that purport to rearrange familiar observations into a new and simpler pattern therefore merit careful consideration. Such is the accelerator hypothesis, proposed by Terry Wilkin [1]. In this issue of Diabetologia, Wilkin provides a brief review of his hypothesis, and explores some of its implications for the classification of diabetes [2].

The accelerator hypothesis proposes that the conditions we call types 1 and 2 diabetes are for practical purposes one and the same. Whilst conceding that the aetiology of beta cell damage may differ, the hypothesis postulates that superimposed insulin resistance then drives both forms of diabetes down the same pathway to end-stage beta cell failure. The difference between the two types of diabetes lies in their rate of progression, which is determined by three accelerators. First comes constitution, for the hypothesis postulates that individuals predisposed to diabetes have an intrinsically high rate of beta cell apoptosis. Even so, diabetes is unlikely to develop unless one or both of the remaining accelerators are encountered. The most important

E. A. M. Gale $(\square)$

Diabetes and Metabolism, Medical School Unit,

Southmead Hospital,

Bristol BS10 5NB, UK

e-mail: Edwin.Gale@bristol.ac.uk of these is insulin resistance. Fuelled by physical inactivity and visceral weight gain, this accelerates beta cell apoptosis and the onset of diabetes. The other accelerator, operative only in those who also have HLA susceptibility alleles or other predisposing genes, is autoimmunity. According to the hypothesis, beta cells stressed by insulin resistance offer more of a target to the immune system, thus superimposing one cause of beta cell death upon another (Fig. 1), which explains why so-called type 1 diabetes progresses more rapidly. Since the major acquired contributor to insulin resistance is excess weight gain, this not only forms the 'missing link' between types 1 and 2 diabetes, but explains why the incidence of both conditions is rising in parallel, and why both are presenting earlier in life [1]. Wilkin's article in this issue of Diabetologia now proposes that the accelerator hypothesis, arguably strengthened by evidence that has emerged over the past 6 years, could form the basis for a new, simpler and more rational classification [2].

Thus the argument. But is it valid? The hypothesis has met with a mixed reception. Brilliantly named and eloquently advocated, it has entered the common parlance of type 1, but not of type 2 diabetes. The original article has been cited 88 times in the literature, and a number of subsequent studies claim to have tested or confirmed it. There has been particular appeal in the notion that increasing adiposity has driven the parallel rise and apparent overlap of types 1 and 2 diabetes in childhood and adolescence. Further support has come from studies that have identified a role for insulin resistance in the pathogenesis of type 1 diabetes, and the hypothesis underpins a proposed prevention trial with metformin. Notwithstanding, opinion is highly polarised: many have dismissed the arguments out of hand, and two referees advised outright rejection of Wilkin's recent article. 
'Double diabetes'

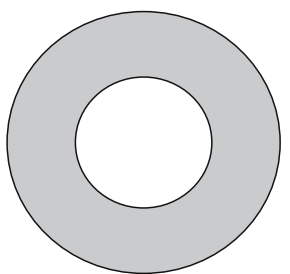

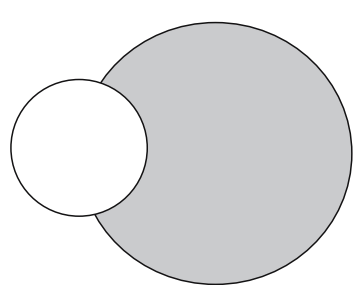

Fig. 1 The accelerator hypothesis postulates that the risk of immunemediated diabetes is superimposed upon the risk of non-immunemediated diabetes. The concept of 'double diabetes' proposes an overlap between the two

Since progress in science depends on dialogue rather than mutual avoidance, and clarity is at all costs better than confusion, my decision was to offer publication, but on the understanding that the article would be accompanied by the critical commentary presented here.

\section{How sound is the rationale?}

A hypothesis should be well-reasoned, consistent with observation, and should generate testable statements that are open to refutation. Let us begin with the rationale. The hypothesis outlines three accelerators - constitution, adiposity and autoimmunity. Adiposity translates into insulin resistance, which translates (in those constitutionally predisposed) into accelerated apoptosis, which translates into autoimmunity in those with genetic susceptibility to this.

Step one: 'Constitution' predisposes to accelerated apoptosis The meaning of this is not clear. People with diabetes undoubtedly have fewer beta cells at diagnosis than those who do not develop diabetes, but we have no idea when beta cell loss begins, or whether there are programmed differences in the intrinsic rate of beta cell loss. And what does "constitution" mean? Most of us would assume that it means much the same as genetic predisposition. If so, the expectation arising from the accelerator hypothesis would be that both forms of diabetes share a common genetic susceptibility to beta cell loss. This, however, is not the case, for not one of the type 2 diabetes genes-TCF $7 L 2$ to take one recent example [3] - has yet been shown to be over-represented in type 1 diabetes. Wilkin responds that this does not disprove the accelerator hypothesis [4], for HLA susceptibility is such a powerful inducer of beta cell loss that other, weaker, genes need not be present in type 1 diabetes. By disposing of one objection, however, he opens the way to another, for the accelerator hypothesis is based upon the claim that both forms of diabetes share a common soil. And what common soil can there be, if there are no genes in common? The observation that the obesity gene FTO is over-represented in type 2 but not type 1 diabetes [5] creates further difficulty for the hypothesis, given that obesity is the proposed link between the two.

Step 2: Insulin resistance is the principal cause of nonimmune diabetes It is wise to be cautious of any statement whose converse is equally true, for insulin secretion and sensitivity are reciprocally related, and it would be just as correct to say that insulin deficiency is the principal cause of diabetes. We should be equally cautious when the term 'insulin resistance' is introduced into discussion without careful qualification, for this is a reliable hint that confused thinking is on its way. Confusion arises when we are seduced into the belief that insulin resistance exists as an objective entity in some platonic universe of its own, whereas it is only a measure of comparison, equivalent to intensity or physical fitness. The terms insulin sensitivity or insensitivity are preferable because they remind us of this. A second reason for confusion is that the term is so often introduced in one context and then used in another. Let us consider some of these contexts before going any further.

The first difference in context is between 'insulin resistance' relative to the body's capacity to make insulin, and 'insulin resistance' relative to that of a healthy population. Used in the first sense, it is quite correct to say that insulin sensitivity determines the level of insulin secretion at which beta cell insufficiency translates into hyperglycaemia, and is therefore the proximate cause of both forms of diabetes. When comparisons are made between test and control populations, however, the conditions need to be carefully defined. For present purposes, the important distinction is between normal and abnormal glucose homeostasis. On current evidence normoglycaemic individuals en route to type 1 diabetes have normal or increased insulin sensitivity [6], whereas sensitivity is decreased in individuals en route to type 2 diabetes. The situation changes as glucose homeostasis is lost, for this involves functional alterations which serve to decrease insulin sensitivity.

Since insulin resistance is a relative term, the important question is 'relative to what?' The unqualified statement that 'type 1 diabetes is associated with insulin resistance' could for example be considered in relation to: (1) the insulin secretory capacity of the body; (2) baseline in the same individual, e.g. as he or she progresses from normal to abnormal glucose tolerance; (3) healthy controls; (4) individuals with type 2 diabetes. The accelerator hypothesis, as may be noted, switches from one to another of these contexts without ever changing gear.

This apart, what is the basis for the proposition that insulin insensitivity accelerates beta cell apoptosis in non-immune forms of diabetes? Here we enter the classic debate as to 
whether insulin insensitivity causes insulin deficiency, or vice versa - the diabetes equivalent of the argument concerning the priority of the chicken or the egg. Given that healthy humans appear able to compensate for an increased workload by increasing their beta cell mass [7], insulin resistance is not a primary cause of insulin deficiency. Investigators therefore seek to explain the origins of diabetes in terms of the resilience, regenerative capacity and/or function of the beta cell $[7,8]$. Once beta cell mass and/or function have become compromised, however, insulin insensitivity assumes an important secondary role. It does not cause beta cell insufficiency, but brings it to light. Metabolic decompensation then triggers a complex sequence of functional changes, some of which-glucolipotoxicity and loss of pulsatile secretion, for example-themselves decrease sensitivity to insulin and promote the familiar selfaugmenting spiral towards progressive beta cell failure.

Step 3: Insulin resistance promotes beta cell autoimmunity The proposal that insulin insensitivity accelerates immunemediated diabetes is based on the assumption that autoimmunity is enhanced by insulin resistance in normoglycaemic individuals, and for this there is no evidence. The frequently quoted observation that GAD expression is increased in cultured human beta cells exposed to increasing concentrations of glucose relates to glucose exposure rather than insulin insensitivity [9], and does not correlate with clinical observation. Autoantibody titres do not rise in parallel with beta cell overload as individuals move from islet autoimmunity to overt diabetes. Furthermore, beta cell rest, as offered for example by supplementation with injected insulin in the diabetes prevention trial-type 1 (DPT-1), had no effect at all upon progression from islet autoimmunity to hyperglycaemia in a large cohort of at-risk relatives [10].

The lack of evidence that insulin insensitivity enhances autoimmunity is matched by a similar lack of evidence that insulin insensitivity precedes diabetes in normoglycaemic individuals - a key prediction of the accelerator hypothesis [1]. Extensive analyses, not available at the time the hypothesis was formulated, now confirm that insulin and/ or C-peptide secretion is similar or slightly reduced in autoantibody-positive first-degree relatives who progress to diabetes compared with non-progressors [6]. Loss of the first-phase insulin response (FPIR) to intravenous glucose precedes and predicts diabetes in first degree relatives with islet autoantibodies [11], and individuals with low FPIR may remain normoglycaemic over months or years [6]. This is because they are very sensitive to insulin. Relative insulin insensitivity will of course determine the time it takes to progress from insulin deficiency to hyperglycaemia [12], but (for the reasons given above) it is misleading to refer to this as 'insulin resistance'.
Three recent studies support the view that insulin sensitivity only becomes an important influence upon progression to hyperglycaemia once critical levels of secretory failure are present. Analysis of progression to diabetes in the oral and parenteral arms of DPT- 1 showed that 31 of 32 individuals tested within 6 months of diabetes onset had an abnormal IVGTT, OGTT, or both [11]. In the parenteral trial, the homeostatic model assessment (HOMA-R) was significantly related to the rate of progress to diabetes only in those with loss of FPIR [11, 13], and the same finding has emerged from analysis of the European Nicotinamide Diabetes Intervention Trial (ENDIT) cohort (unpublished results). The size of these studies allows us to conclude with some confidence that insulin sensitivity is not a major influence upon progression to diabetes in those with FPIR above the tenth centile of a control population; its influence only emerges in those with advanced insulin secretory failure or early metabolic abnormalities. Independently, and by a different route, analysis of a subset of participants in the SEARCH study led to a similar conclusion: increasing BMI is associated with decreasing age of diagnosis of type 1 diabetes only in those with evidence of reduced beta cell function [14]. We may conclude that decreasing insulin sensitivity can accelerate onset in those with advanced beta cell failure and/or metabolic decompensation, but has no causal role in the pathogenesis of type 1 diabetes.

\section{Does the hypothesis fit the facts?}

Is the accelerator hypothesis therefore beyond salvage? Not entirely. Its strength lies in the fact that accelerators are undoubtedly present. Few would doubt, for example, that earlier onset of non-immune diabetes is in some way linked to the rise of childhood obesity, and insulin insensitivity is the most plausible mechanism for this. There is compelling evidence that type 1 diabetes has shown a similar left-shift towards earlier onset [15], and it is entirely reasonable to believe that hyperglycaemia will be expressed earlier (i.e. at a higher level of beta cell mass and/or function) in an insulin-insensitive individual than in a more sensitive one; progress from incipient metabolic dysfunction to overt diabetes is also likely to be accelerated. Wilkin has thus introduced a useful concept into the debate about type 1 diabetes. But is it correct to assume that childhood obesity is the only accelerator of type 1 diabetes?

Increased growth is the common factor linking a wide range of observations. On aggregate, children who go on to develop diabetes grow more rapidly, even before birth [16], and increased linear growth precedes the onset of childhood diabetes. Priscilla White noted in 1932 that children with diabetes were on average $5.6 \mathrm{~cm}$ above their expected 
height at the time of diagnosis [17]. Measures of height and weight at or shortly after diagnosis of diabetes may of course be affected by the associated metabolic disturbance, and reliable estimates of prediabetic growth therefore require accurate growth records from birth.

The first large study to meet this requirement took advantage of the Swedish national registry to evaluate antecedent growth in children diagnosed in 1985-1986 and population controls. Boys with diabetes were significantly taller than their controls, with a similar trend for girls, but weight-for-height was not increased in either sex. This study thus indicated that linear growth, and weight (but not obesity), were the risk factors associated with diabetes [18]. A subsequent Swedish study considered children diagnosed from 1974 to 1988 . Length at birth did not differ, although probands were slightly taller at 18 months; risk of progression to diabetes was associated with increased weight gain up to the age of 30 months [19]. A study from Finland confirmed previous associations with increased height and weight in children diagnosed from 1986 to 1989, and also identified obesity as an independent risk factor; a relative weight $>120 \%$ after 3 years of age more than doubled the risk of progression to diabetes [20]. It is tempting to speculate that BMI has only recently become important as a risk factor for type 1 diabetes, in parallel with the rise of childhood obesity, but there is no way of being sure about this.

Other studies have been based on measures of height and weight following diagnosis; these rely on population reference data for their conclusions [21-24]. Some have purported to test or confirm the accelerator hypothesis, but a hypothesis cannot be tested by repeating the observations upon which it was originally based. The best and largest of these studies makes no such claim, but confirms that height standard deviation score (SDS) was higher in recently diagnosed children than in reference populations. BMI SDS was also consistently greater during the 14 year study, rose over the study period, and was inversely related to age at onset [24].

We should not forget that, although highly significant on statistical analysis, the difference between patients and controls is not that great in absolute terms. For example, patients diagnosed between the ages of 15 and 20 years in the study of Knerr et al. had BMIs of $21.6 \pm 0.13 \mathrm{~kg} / \mathrm{m}^{2}$ compared with $20.6 \pm 0.02 \mathrm{~kg} / \mathrm{m}^{2}$ (mean $\pm \mathrm{SEM}$ ) in the reference population [24]. The overall contribution of height, weight and BMI to the risk of childhood type 1 diabetes has been estimated at about $20 \%$ [25].

\section{Conclusions}

I do not believe the accelerator hypothesis, at least as presently formulated. The concept is ingenious but, in common with other universal explanations, it ends by explaining very little.
The underlying rationale, although persuasively argued, skates over a number of fundamental objections. There is no evidence to support the common soil hypothesis. There is no evidence that insulin insensitivity accelerates beta cell apoptosis in normoglycaemic individuals at risk of type 2 diabetes, although it may have important secondary effects as beta cell function begins to fail. There is no evidence that insulin secretion is increased during the type 1 diabetes prodrome, and there is no evidence that, if present, insulin insensitivity would accelerate beta cell autoimmunity. Children destined to develop type 1 diabetes grow faster and fatter in early life, but this could be a consequence rather than a cause of their predisposition to diabetes. Childhood obesity may have contributed to the linear rise of childhood type 1 diabetes over the past 50 years, but does not explain it. Features shared by types 1 and 2 diabetes in adolescence are more convincingly explained by overlap than by overlay'double' rather than single diabetes (Fig. 1). Nor does the contention that adolescent-onset types 1 and 2 diabetes share a common background explain why they affect different ethnic populations.

So can the accelerator hypothesis provide a rationale for intervention with metformin to prevent type 1 diabetes? It can, but the rationale is not unique to the hypothesis: the concept of 'double diabetes' could justify the same intervention. Is the rationale convincing? No, given the lack of evidence that an insulin-sensitising agent such as metformin will have any effect upon immune-mediated beta cell destruction. Yes, if you wish to slow progression from the first signs of metabolic decompensation to overt hyperglycaemia, although (by analogy with type 2 diabetes) intensive lifestyle intervention might prove as effective. Intervention at this late stage could postpone the need for insulin by a few months, and this might be useful as an adjunct to other interventions. It is unlikely to influence the course of the underlying disease.

Finally, and despite its limitations, the accelerator hypothesis has introduced some valuable concepts into the debate surrounding type 1 diabetes. This Editorial has been written in the belief that these core concepts are of real novelty and interest, but only when dissected away from broader and less profitable speculation. There are indeed accelerators for type 1 diabetes, and increasing obesity is likely to assume increasing importance in the years to come. Other accelerators, however, whether genetic, metabolic or immune, are also likely to be present [15]. These, in my view, are fruitful areas for investigation and debate - and could in time lead to the rebirth of the accelerator hypothesis in a new guise.

\section{References}

1. Wilkin TJ (2001) The accelerator hypothesis: weight gain as the missing link between type I and type II diabetes. Diabetologia 44:914-922 
2. Wilkin TJ (2007) Changing perspectives in diabetes: their impact on its classification. Diabetologia DOI 10.1007/s00125-007-0665-5

3. Field SF, Howson JMM, Smyth DJ, Walker NM, Dunger DB, Todd JA (2007) Analysis of the type 2 diabetes gene, TCF7L2, in 13,795 type 1 diabetes cases and control subjects. Diabetologia 50:212-213

4. Wilkin TJ (2007) The accelerator hypothesis cannot be tested using the type 2 diabetes gene TCF7L2. Diabetologia DOI 10.1007/s00125-007-0725-x

5. Field S, Howson J, Hafler J et al (2007) Analysis of the obesity gene FTO in 14,803 type 1 diabetes cases and controls. Diabetologia (in press)

6. Sherry NA, Tsai EB, Herold KC (2005) Natural history of $\beta$-cell function in type 1 diabetes. Diabetes 54(Suppl 2):S32-S39

7. Prentki M, Nolan CJ (2006) Islet $\beta$-cell failure in type 2 diabetes. J Clin Invest 116:1802-1812

8. Donath MY, Ehses JA, Maedler K et al (2005) Mechanisms of $\beta$ cell death in type 2 diabetes. Diabetes 54(Suppl 2):S108-S113

9. Björk E, Kämpe O, Karlsson FA et al (1992) Glucose regulation of the autoantigen $\mathrm{GAD}_{65}$ in human pancreatic islets. J Clin Endocrinol Metab 75:1574-1576

10. Diabetes Prevention Trial-Type 1 Diabetes Study Group (2002) Effects of insulin in relatives of patients with type 1 diabetes mellitus. N Engl J Med 346:1685-1691

11. Barker JM, McFann K, Harrison LC et al (2007) Pre-type 1 diabetes dysmetabolism: maximal sensitivity achieved with both oral and intravenous glucose tolerance testing. J Pediatr 150:31-36

12. Fourlanos S, Narendran P, Byrnes GB, Colman PG, Harrison LC (2004) Insulin resistance is a risk factor for progression to type 1 diabetes. Diabetologia 47:1661-1667

13. Tsai EB, Sherry NA, Palmer JP, Herold KC (2006) The rise and fall of insulin secretion in type 1 diabetes mellitus. Diabetologia 49:261-270

14. Dabelea D, D'Agostino RB, Mayer-Davis EJ et al (2006) Testing the accelerator hypothesis. Body size, $\beta$-cell function, and age at onset of type 1 autoimmune diabetes. Diabetes Care 29:290-294
15. Gale EAM (2005) Spring harvest? Reflections on the rise in type 1 diabetes. Diabetologia 48:2445-2450

16. Dahlquist G, Bennich SS, Källén B (1996) Intrauterine growth pattern and risk of childhood onset insulin dependent (type 1) diabetes: population-based case-control study. BMJ 313:1174-1177

17. White P (1932) Diabetes in childhood and adolescence. Lea and Febiger, Philadelphia, PA, p 105

18. Blom L, Persson LÅ, Dahlquist G (1992) A high linear growth is associated with an increased risk of childhood diabetes mellitus. Diabetologia 35:528-533

19. Johansson C, Samuelsson U, Ludvigsson J (1994) A high weight gain early in life is associated with an increased risk of type I (insulin-dependent) diabetes. Diabetologia 37:91-94

20. Hyppönen E, Virtanen SM, Kenward MG, Knip M, Åkerblom HK; The Childhood Diabetes in Finland Study Group (2000) Obesity, increased linear growth, and risk of type I diabetes in children. Diabetes Care 23:1755-1760

21. Kibirige M, Metcalf B, Renuka R, Wilkin TJ (2003) Testing the accelerator hypothesis: the relationship between body mass and age at diagnosis of type 1 diabetes. Diabetes Care 26:2865-2870

22. Betts P, Mulligan J, Ward P, Smith B, Wilkin T (2005) Increasing body weight predicts the earlier onset on insulin-dependent diabetes in childhood: testing the 'accelerator hypothesis' (2). Diabet Med 22:144-151

23. Kordonouri O, Hartmann R (2005) Higher body weight is associated with earlier onset of type 1 diabetes in children: confirming the 'accelerator hypothesis'. Diabet Med 22:1783-1784

24. Knerr I, Wolf J, Reinehr T et al; on behalf of the DPV Scientific Initiative of Germany and Austria (2005) The 'accelerator hypothesis': relationship between weight, height, body mass index and age at diagnosis in a large cohort of 9,248 German and Austrian children with type 1 diabetes mellitus. Diabetologia 48:2501-2504

25. Dahlquist G (2006) Can we slow the rising incidence of childhood-onset autoimmune diabetes? The overload hypothesis. Diabetologia 49:20-24 